\title{
How Single-Molecule Localization Microscopy Expanded Our Mechanistic Understanding of RNA Polymerase II Transcription
}

\author{
Peter Hoboth ${ }^{1,2}$, Ondřej Šebesta ${ }^{2}$ and Pavel Hozák 1,3 * \\ 1 Department of Biology of the Cell Nucleus, Institute of Molecular Genetics of the Czech Academy of \\ Sciences, Vídeňská 1083, 14220 Prague, Czech Republic \\ 2 Faculty of Science, Charles University, Albertov 6, 12800 Prague, Czech Republic \\ 3 Microscopy Centre, Institute of Molecular Genetics of the Czech Academy of Sciences, Vídeňská 1083, \\ 14220 Prague, Czech Republic \\ * Author to whom correspondence should be addressed.
}

\begin{abstract}
Classical models of gene expression were built using genetics and biochemistry. Although these approaches are powerful, they have very limited consideration of the spatial and temporal organization of gene expression. Although the spatial organization and dynamics of RNA polymerase II (RNAPII) transcription machinery has fundamental functional consequences for gene expression, its detailed studies have been for long time abrogated by the limits of classical light microscopy. The advent of super-resolution microscopy (SRM) techniques allowed for the visualization of the RNAPII transcription machinery with nanometer resolution and millisecond precision. In this review, we summarize the recent methodological advances in SRM, focus on its application for studies of the nanoscale organization in space and time of RNAPII transcription, and discuss its consequences for the mechanistic understanding of gene expression.
\end{abstract}

Keywords: cell nucleus; gene expression; transcription foci; transcription factors; super-resolution microscopy; structured illumination; stimulated emission depletion; stochastic optical reconstruction; photoactivation

\section{Introduction}

The genome is a complex and very dense viscoelastic polymer matrix and therefore it is difficult to study individual components of the gene expression machinery with conventional light microscopy. Optical resolution of the conventional light microscopy is limited by the diffraction of light and allows to distinguish the objects only if they are $\sim 200$ $\mathrm{nm}$ apart. This $\sim 200 \mathrm{~nm}$ resolution limit of the classical light microscope is given by the nature of light and results from the fundamental laws of physics. Ernst Abbe postulated in 1873 that the limit of discrimination will never pass significantly beyond half the wavelength of blue light $[1,2]$. According to the Abbe's law of the diffraction limit, the best achievable spatial resolution in the object plane $\left(d_{x, y}\right)$ and along the optical axis $\left(d_{z}\right)$ is $d_{x, y}=\lambda / 2 n \sin \alpha$ (1) and $d_{z}=2 \lambda / n \sin \alpha$ (2), resp. In (1) and (2) $\lambda$ represents the wavelength in vacuum of the light used for imaging and $n \sin \alpha$ represents the numerical aperture (NA) of the microscope objective lens, where $n$ is the refractive index of the sample and $\alpha$ is a half of the objective lens aperture angle. Hence, reducing the wavelength of the light used for imaging and/or increasing the NA of the objective lens improves the spatial resolution of a conventional light microscope. Lord Rayleigh elaborated on the diffraction limit and in 1896 postulated that the smallest resolvable distance $\left(d_{\min }\right)$ between two points is proportional to the wavelength in vacuum of the light used for imaging and inversely proportional to the NA with a factor of 0.61 [3], which is expressed by the formula $d_{\min }=0.61 \lambda / N A$ (3). According to (3) it is possible to improve the $d_{\min }$ 
by shortening the $\lambda$ and therefore use of electrons instead of light allowed for the visualization of the biological specimens at the ultrastructural level. Many important discoveries of the ultrastructural organization of the eukaryotic cell nucleus, such as the precise localization of transcribed DNA [4] or the ultrastructure of transcription sites [5] were possible thank to the electron microscopy. However, more detailed summary of the functional organization of the cell nucleus as revealed by EM is beyond the scope of this review and were reviewed elsewhere [6, 7]. Very specific and invasive sample preparation is however the limitation of electron microscopy and renders it impossible for live-cell application.

Super-resolution microscopy (SRM), in contrary to the electron microscopy, allows for live-cell imaging at previously unprecedented spatial and temporal level. Novel and progressive SRM approaches helped to uncover many previously undetected dynamic mechanisms that regulate mammalian gene expression. Although the term gene expression has broad implications, from chromatin organization in space and time to the export of mRNA from the nucleus for the translation into the proteins in the cytosol, in this review we use the term gene expression for RNA polymerase II (RNAPII) mediated transcription. Nevertheless, RNAPII is not a solo player but its function is related to the overall gene expression processes and also intimately linked with the steps preceding as well as succeeding the transcription. Although in this short review we will introduce SRM techniques in general, we will put emphasis on the single-molecule localization microscopy (SMLM) and present the variety of fluorophores suitable for SMLM. Then we will first focus on SMLM applications to studying the dynamics of transcription factors and finally on the RNAPII itself.

\section{Brief Introduction to SRM}

Although Abbe postulated that the limit of discrimination is given by a half the wavelength of blue light, he also acknowledged that this limit could be surpassed by applications based on principles that are outside of the law that he postulated [1,2]. Fluorescent far field optical microscopy allows detection of light at many wavelengths away from the sample and is currently the basis of the most biological applications of SRM [8-10]. SRM achieves sub-diffraction limited resolution by time-controlled emission of fluorescence from only a subset of fluorophores from the total fluorophore population in the specimen. Two main concepts of SRM are (i) reversible saturable optical fluorescence transitions (RESOLFT) that allow deterministic temporal control of fluorophore emission and (ii) single-molecule localization microscopy (SMLM) based on the stochastic temporal control of fluorophore emission $[2,11,12]$.

\subsection{Deterministic SRM Approaches: SIM and STED}

The RESOLFT family $[8,9,13,14]$ includes saturated structured illumination microscopy (S-SIM) [15], which is an upgrade of previous structured illumination microscopy (SIM) [16]. In the classical SIM Gustafsson implemented high-frequency line-patterned illumination and improved axial and lateral resolution by a factor of two [16, 17]. The subsequent 3D SIM improved spatial resolution by additional factor of two [18-20]. However, classical SIM did not demonstrate the potential to increase the resolution without limit and therefore the classification of SIM as a typical SRM varies [10-12]. Nevertheless, S-SIM, which is a nonlinear modification of classical SIM, is in theory capable of unlimited resolution [15] and is also applicable for live-cell imaging [21]. A typical representative of the RESOLFT family is stimulated emission depletion (STED) microscopy and its variations [22]. The resolution of a confocal microscope, on which STED is typically based, is increased by attenuating the fluorescence at the periphery of the excitation spot. The fluorescently labeled sample is illuminated by excitation laser simultaneously with the depletion laser that has wavelength within the emission spectrum of the imaged fluorophore. The depletion laser beam has a ring or donut shape and is superimposed over the excitation laser beam. The depletion laser at certain intensity, called saturation intensity, then switches of the fluorescence over controlled area at the periphery of the excitation spot by a process called stimulated emission [23] and effectively reduces the diameter of a recorded fluorescence spot. Figure 1 shows visual comparison of histone H2B tagged 
with SNAP-tag and labeled in living U-2 OS cells with the SNAP substrate JF549, followed by the indirect immunofluorescence labeling of the RNAPII in the same cells upon chemical fixation and imaged by SIM, STED and ASTORM. SMLM technique ASTORM is introduced in the next section followed by the section on the fluorescent labeling strategies for SMLM.

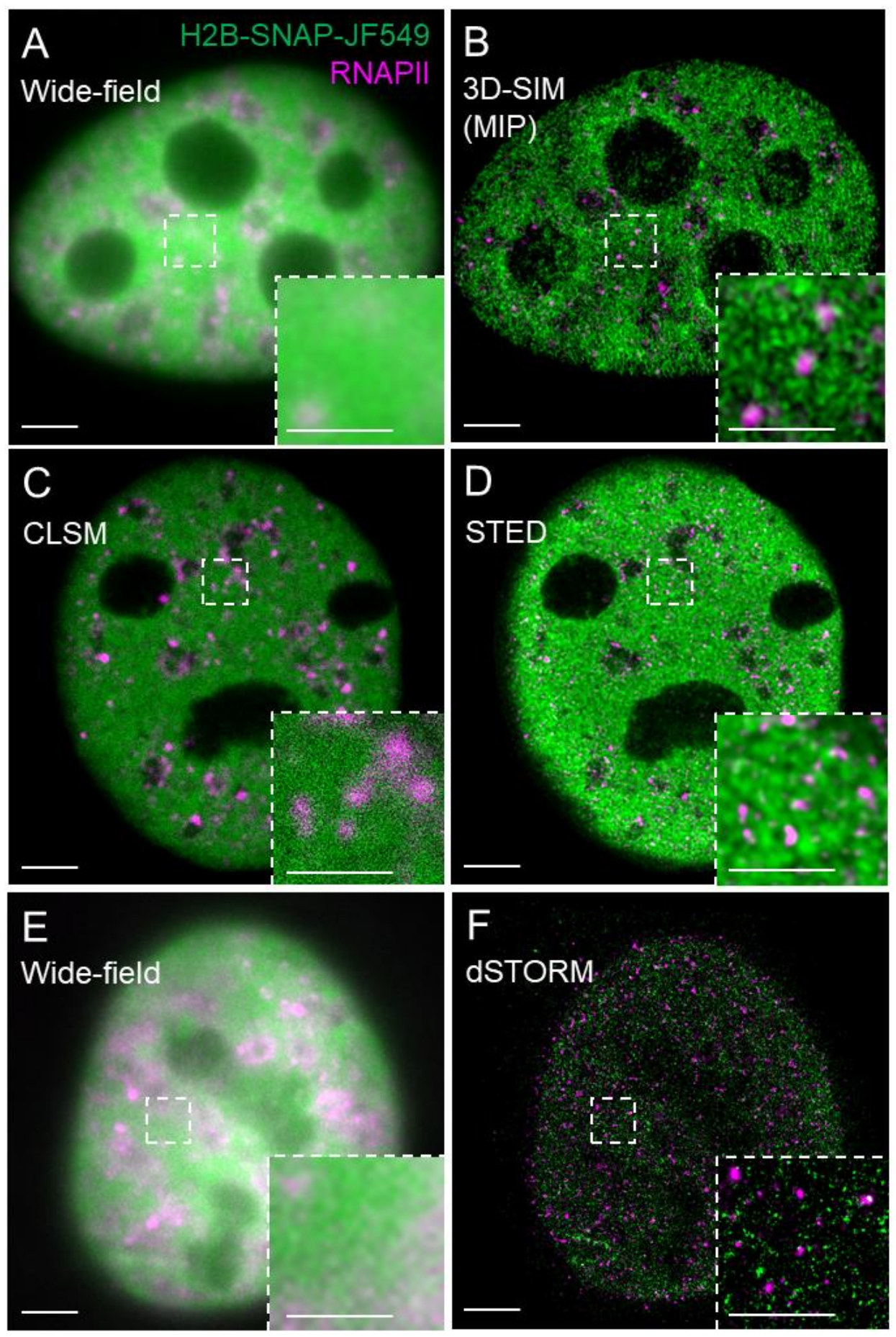

Figure 1. Visual comparison of SIM, STED and dSTORM. Nuclei of U-2 OS cells expressing histone H2B tagged with SNAP-tag were labeled with the JF549 SNAP substrate (H2B-SNAP-JF549, green), chemically fixed and indirectly immunolabeled against RNAPII with a secondary antibody conjugated with Alexa Fluor 647 (magenta, Abberior STAR 635P for STED). The same cell was imaged by (A) wide-field and (B) 3D-SIM (MIP - maximum intensity projection of a 3D-SIM zstack); (C) confocal laser scanning microscopy (CLSM) and (D) STED microscopy followed by deconvolution; (E) wide-field and (F) 2D dSTORM. Bar $=5 \mu \mathrm{m}$; inset $1 \mu \mathrm{m}$. 


\subsection{Stochastic SRM Approaches: Single-Molecule Localization Microscopy}

SMLM, in contrast to the above-discussed deterministic SRM techniques, allows for the visualization of individual fluorophores at the level of individual molecules. This revolutionary approach is based on the temporal separation of individual fluorescent molecules based on a relatively simple principle that allows for the detection at subsequent time intervals of a sparse subset of well-separated fluorophores and reconstruction of a final super-resolved image from the localization of the centers of the fluorophores (Fig. 2). SMLM techniques originally included photoactivated or fluorescence photoactivation localization microscopy (PALM) [24] or FPALM [25] and stochastic optical reconstruction microscopy (STORM) [26] or direct STORM (dSTORM) [27-29] and their modifications. These methods limit the number of fluorophores that emit photons at the same time and assemble the final super-resolved image from sequentially emitting fluorophores. Reduced probability of simultaneous detection of overlapping signals in one acquisition frame allows to localize the center of individual emitters with the precision in the range of lower tens of nanometers, depending on the number of emitted photons. PALM utilizes the photo-activatable fluorescent proteins (PA-FPs) that can be reversibly switched between the non-fluorescent and fluorescent states or the photo-convertible FPs (PC-FPs) that can be switched between two fluorescent states with the different excitation and emission pattern [30,31]. Individual molecules of the PA- and PC-FPs are stochastically photoactivated and photo-converted, resp., while neighboring molecules remain non-fluorescent or undetected and therefore individual FP molecules can be sequentially localized at different time intervals. Fluorescence emitted by the individual and spatio-temporaly well separated PA or PC form of a FP is detected by sensitive cameras and fitting of the Gaussian profile to the signal enables to measure the position of a molecule with a precision far better than in the diffraction limited conventional fluorescence microscopy. The principle of reversible stochastic photo-switching of organic fluorochromes between the fluorescent and long-lived dark states was first utilized in STORM using photo-switchable dye pairs [26]. This principle allowed for the detection of a sparse subset of fluorochromes in their fluorescent "on" state that are well separated at given time-point. Thus, the precise localization, e.g. center of the mass, of individual particles can be determined with high precision and this process is iteratively repeated (Fig. 2A). Final super-resolved image is created by the sum of individual single molecule localizations (Fig. 2B-D) and therefore resembles of artistic painting technique pointillism (Fig. 2E-G) [32]. Soon after its introduction, STORM was implemented for 3D [33, 34] and live-cell imaging [35]. In contrast to PALM and STORM that use PA-FPs and combination of activator and reporter pairs of organic fluorochromes, resp., dSTORM uses conventional fluorescent probes such as labeled antibodies or self-labeling tags [27, 29]. 

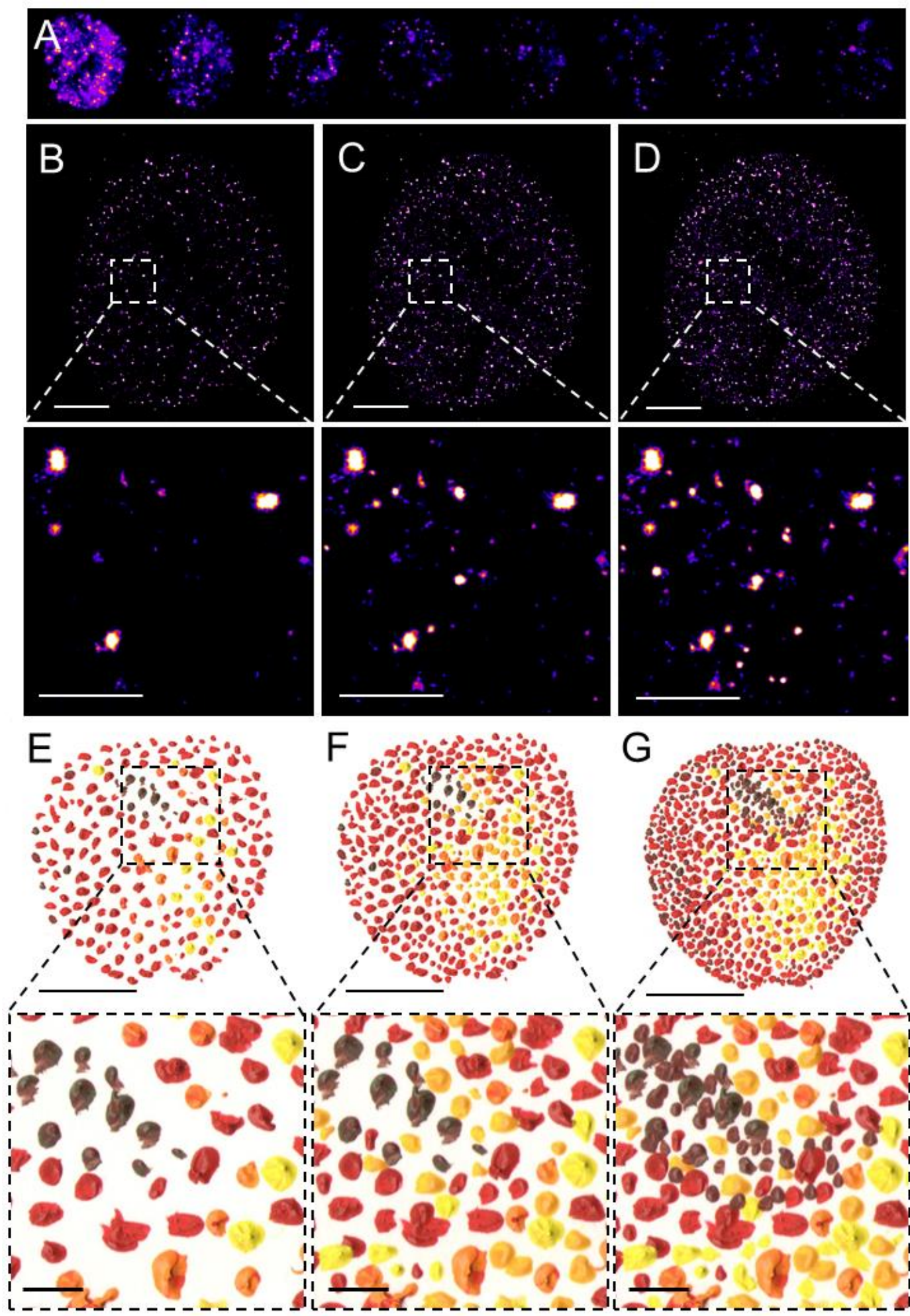

G
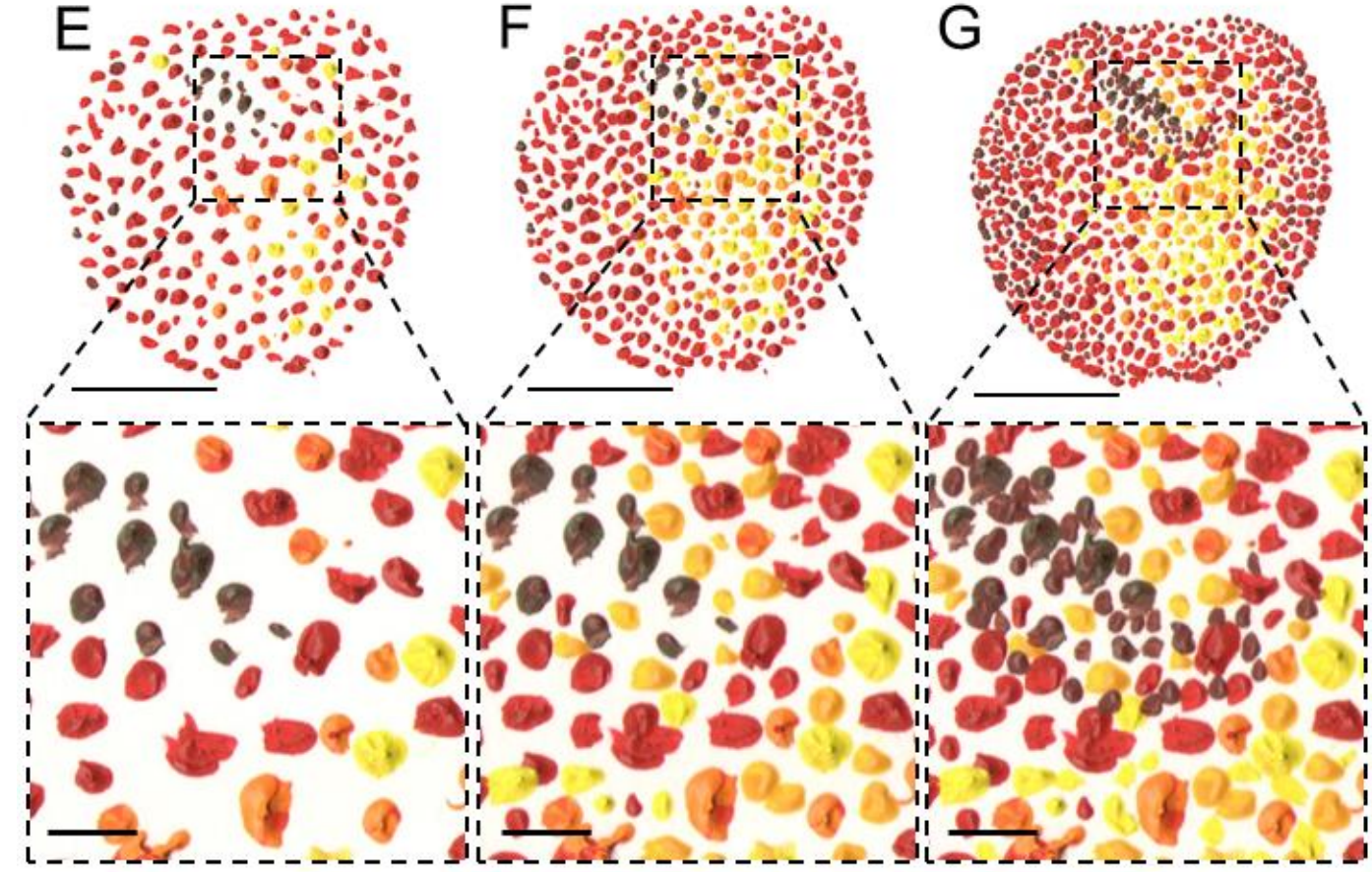

Figure 2. Pointilistic nature of single-molecule localization microscopy. (A) Individual frames from the time-lapse acquisition of raw blinking of Alexa Fluor (AF)647-labeled secondary antibody against primary anti-RNAPII antibody (RNAPII-AF647). The signal of individual AF647 molecules accumulates in time $(B, C)$ to create the final dSTORM image of RNAPII (C). The imaging process in (B-D) resembles artistic painting style called pointillism in which accumulation of color spots on a canvas (E, F) creates final painting (G). Artistic paintings in (E-G) were kindly provided by Katarína Mrvová. White bar $=5 \mu \mathrm{m}$; inset $1 \mu \mathrm{m}$. Black bar $=5 \mathrm{~cm}$; inset $1 \mathrm{~cm}$.

SMLM along the optical plane (z-axis) is more challenging than in the imaging $(x, y)$ plane for several reasons [11,36]. First, out-of-focus fluorescence from above and below the focal plane precludes the precise localization of fluorophores. Second, bleaching of the 
fluorophores outside of the focal plane reduces localization density. Third, the number of molecules required to adequately sample a given spatial frequency scales exponentially with the spatial dimension and thus making 3D imaging more challenging than 2D imaging. Solutions for 3D SMLM with improved localization accuracy along the z-axis utilize astigmatism [33, 34], double-plane detection [37] or engineered PSF with the double-helix shape [38].

Wide-field (WF) illumination is commonly used in SMLM but suffers from significant drawbacks [39], such as is the first and second limitation of 3D SMLM mentioned just above. Elimination of those drawbacks significantly improved the single molecule localization accuracy. Total internal reflection fluorescence (TIRF) microscopy exploits the exponential decay of the evanescent wave created by the complete reflection under the angle larger than the critical angle of a laser beam from the glass surface at the interface between glass surface and the sample [40]. Thus, the evanescent wave selectively illuminates a region above the glass surface that is up to $200 \mathrm{~nm}$ into the specimen. Highly inclined and laminated optical sheet (HILO) allows for selective illumination of thin regions deeper than TIRF angle [41]. In contrast to TIRF, HILO uses a laser beam with an incident angle slightly below the critical angle, which creates a slightly inclined light sheet through the specimen, but just above the glass surface. HILO illumination increases specific intensity, greatly reduces background and thereby allows for the precise visualization and quantification of single molecule dynamics in living cells. More technically demanding modalities for selective plane illumination, such as Gaussian light sheet (LS), require additional illumination objective, which creates thin sheet of illumination perpendicular to the detection objective [42]. LS illumination permits imaging of thick samples but has limited use for SMLM. Recent combination of LS with the above discussed RESOLFT neutralized the resolution-limiting role of diffraction in LS. LS-RESOLFT is therefore conceptually diffraction-unlimited and has a potential to develop toward molecular-scale resolution [43]. Bessel beam selective plane (BBSP) illumination overcomes the limitations of Gaussian light sheet and allows for the fast imaging across large volumes with increased single molecule localization precision due to the reduced background fluorescence [44, 45]. Lattice light sheet (LLS) uses ultrathin light sheets derived from 2D optical lattices that are scanned through the specimen to image across large volumes with high spatial and temporal resolution and minimal photo-damage [46]. In the reflected light sheet (RLS) microscopy a mirror reflects the elliptical laser beam and thereby creates a thin sheet of light parallel with the imaging plane, which allows for the selectively optical sectioning $(<0.5$ um) throughout the cell nucleus. Selective illumination of $<0.5 \mu \mathrm{m}$ plane results in the photoactivation of only a small subset of photoactivatable fluorescent proteins and in turn dramatically increases the signal-to-noise ratio critical for the direct monitoring of the single-molecule kinetics [47, 48].

SMLM and STED has recently met at their intersection called minimal photon emission fluxes (MINFLUX). This novel concept combines stochastic switching of the fluorophores on and off as in PALM or STORM but the emitter is localized by a doughnutshaped excitation beam that is used in STED [49]. In result, MINFLUX and its derived fluorescence nanoscopies require up to orders of magnitude fewer photon emissions to deliver single-digit nanometer spatial resolution and 100-times improved temporal resolution as compares to its parent imaging approaches [50]. MINFLUX and its modifications are applicable in living cells for 3D multicolor imaging and microsecond tracking [51, 52] and awaits further explorations.

In this review we will further discuss the developments of the fluorescent labeling strategies for SMLM and then their use together with the above reviewed SRM techniques to revealed the novel paradigms in the regulation and dynamics of RNAPII transcription.

\section{Progressive Development of Fluorophores for SMLM}

Rapid development of live-cell SMLM applications is linked to the progressive development of fluorophores with specific and unprecedented photophysical properties. Fluorophores used in SMLM can either be genetically encoded FPs, "self-labeling" tags 
such as HaloTag or SNAP that covalently associate with organic fluorochromes or organic fluorochromes such as Alexa Fluores attached directly to a primary antibody or attached to a secondary antibody directed against the primary antibody as in classical immunofluorescence protocols (Fig. 1 and 2). A plethora of conventional fluorophores, such as Alexa Fluor 647 that is the most commonly used in dSTORM [35, 53], are suitable for SMLM [39]. The advantage of rhodamine-based fluorophores is that they exist in a dynamic equilibrium between a fluorescent zwitterion and a non-fluorescent but cell-permeable spirocyclic form. This feature of rhodamine dyes and their derivatives can be differently utilized in various SRM modalities that have different requirements of the dynamic equilibrium between the zwitterion and spirocyclic rhodamine forms [54, 55].

As mentioned above, individual fluorescent proteins are randomly photo-activated or photo-converted at different time-points in PALM, while their neighbors remain dark or undetected $[24,25,30]$. The monomeric members of Eos family of the PC-FPs [54] provide high brightness with good contrast and are suitable for live-cell SRM [24, 30]. Photoconvertibe fluorescent protein mEos2 [57] is an improvement of its parent protein mEosFP that was not suitable for use in mammalian cells due to its maturation at lover temperatures. Utilization of mEos2 facilitated for instance the single-molecule kinetics study of transcription factors (TFs) glucocorticoid receptor (GR) and estrogen receptor- $\alpha$ (ER) [47]. Photo-convertibe fluorescent protein Dendra2 [58] is initially green-emitting and upon $405 \mathrm{~nm}$ illumination converts into a red-emitting form [57, 58]. Illumination with the very low $\left(\sim 1 \mathrm{~W} / \mathrm{cm}^{2}\right)$ intensity of $405 \mathrm{~nm}$ light allows for the photo-conversion of only a small subset of Dendra2 molecules in the sample. Favorable photo-physics [59] and low aggregation propensity [62] of Dendra2 made it optimal to study for instance the dynamics of RPB1, the large catalytic subunit of RNAPII [63-65], transcription coactivator Mediator [66] or TFs c-Myc and P-TEFb [67]. However, the photo-converted Dendra2 molecules remain in the red emitting state for several tens or even hundreds of milliseconds and therefore a single molecule may appear in multiple acquisition frames. Moreover, in addition to irreversible photo-bleaching, Dendra2 molecules undergo intermittent photophysical blinking transitions $[61,68]$ that obscure direct correlation between counts of detections and exact numbers of molecules [64].

Genetically encoded self-labeling protein tags such as HaloTag and SNAP [69-71] have enzymatic activity through which covalently attach cell permeable fluorescent organic dyes that can be also photoactivable. HaloTag and SNAP tagged proteins exhibited stable binding events in the nucleus [67] but these unspecific binding events were separated from the specific binding events of the proteins of interest [72]. A comparative SRM study of tagged TF favored HaloTag in terms of unspecific binding, photostability of the conjugated fluorophore or localization precision [73]. Discovery of a fine-tuning method for the development of new and highly photostable fluorophores called Janelia Fluors (JFs) [74] for live cell SRM revolutionized the field and opened almost unlimited opportunities to explore living systems at high spatial and temporal resolution. JFs are available as reactive organic molecules for direct labelling or as HaloTag and SNAP substrates. Important advantage of the many of the JFs is their fluorogenicity, e.g. significant increase in fluorescence upon their binding to HaloTag or SNAP, which alleviates the necessity of extensively washing out unbound fluorophores and reduces unspecific background [75]. Some of the most frequently used JFs so far (Tab. 1) were JF549, which is a prototypical JF and a direct analog of TMR or its silicone-containing far-red counterpart JF646 [74, 76]. Photoactivatable versions of JF549 and JF646 allows to switch on only a subset of fluorophores, which is advantageous for single-particle tracking (SPT) in living cells [77].

Development of PA fluorochromes extended the possibilities previously offered by PA- or PC-FPs. However, the dependence on the photoactivation for SMLM and SPT can be genetically circumvented by the precise copy number control of the fluorescently labelled fusion proteins [78]. This approach is particularly useful for the imaging of individual molecules in the densely packed nucleus. Progressive development of finely-tuned fluorophores [55] including fluorophores with very specific features, such as photo- 
senzitizers that generate reactive oxygen species [79] for delicate applications and allowing for multiplexing at high spatial and temporal resolution [54] continues to push the boundaries of SMLM applications. Moreover, the fluorescent signal of individual molecules of interest can be amplified by multivalent tags such as Sun-tag [80] or repetitive HaloTag [71, 78].

Taken together, rapidly-growing selection of highly specific fluorescent markers with superb properties binding to or being expressed as a part of individual molecules makes SMLM the method of choice to study the functional architecture of the cell nucleus and dynamics of gene expression within its spatial context. Some of the key applications of fluorophores for SMLM studies of the RNAPII transcription and associated molecular processes are summarized in Tab. 1. We will further discuss in the following sections how SMLM expanded our understanding of RNAPII transcription using several studies of TF and RNAPII dynamics as examples.

Table 1. Selected exemplar SMLM approaches of tagged TFs and RNAPII in model systems.

\begin{tabular}{ccccc}
\hline Imaging approach & Utilized fluorophore & Target molecule & Model system & \multicolumn{2}{c}{ Reference } \\
\hline HILO, SPT & 3xHaloTag-JF549 & Sox2 & mESCs & {$[76]$} \\
\hline BBSP 2D SPT & HaloTag-TMR & Sox2, Oct4 & mESCs & {$[70]$} \\
MFM 3D SPT & Dendra2 & c-Myc, P-TEFb & U-2 OS cell line & {$[65]$} \\
\hline PALM, SPT & HaloTag-JF549 & Sox2 & mESCs & {$[90]$} \\
\hline LLS, SPT, PCC & mEos2 & GR & MCF-7 cell line & {$[45]$} \\
\hline RLS, RT & Halo-JF549/646 & Med & mESCs & {$[64]$} \\
\hline RLS SRM & Dendra2 & RPB1 & mESCs & {$[90]$} \\
\hline \multirow{2}{*}{ PALM } & HaloTag-JF549 & Sox2 & U-2 OS cell line & {$[61]$} \\
\hline \multirow{2}{*}{ tcPALM } & Dendra2 & RPB1 & MEFs & {$[62,63]$} \\
\hline tcPALM & Halo-JF549/646 & RPB1 & mESCs & {$[64]$} \\
\hline SPA-SPT & Halo-PA-JF549 & RPB1 & U-2 OS & {$[101]$} \\
\hline
\end{tabular}

\section{Revised Model of Transcription Pre-Initiation Based on the TF Single Molecule Kinetics}

Diffusion of TFs allows them to search for their targets to initiate the transcription of specific genes and thereby regulate patterned gene expression [81-83]. Earlier studies of the TF diffusion, mostly performed using fluorescence recovery after photobleaching and similar methods [84], enabled quantification of the dynamics of TF subpopulations but underestimated the impact of nuclear architecture on TF dynamics and did not reveal the DNA binding kinetics of individual TF molecules [85-87]. Implementation of SMLM for the investigation of the TF dynamics highlighted that the interaction of regulatory proteins with target sites in chromatin is even more dynamic process than previously thought, which led to the revision of the model in which the pre-initiation complex assembles by subsequent recruitment of its individual components [88].

Several TFs were recently scrutinized by SMLM, which helped to uncover the common as well as distinct dynamic patterns, explaining the universal mechanisms as well as the roles of distinct TFs in distinct transcriptional programs. In general, the TF kinetics at single-molecule level was inferred either by fast time-lapse imaging and SPT [89, 90] or by blurring off the fast diffusing molecules and localization of residing TFs at long acquisition times. Stochastic labeling and long-time SPT of JF549-labelled Sox2 tagged with 3repeats of HaloTag and imaged in living mESCs by time-lapse microscopy using HILO illumination revealed that Sox2 dynamically hops and interacts locally in the nucleus [78]. This study underscored that functional architecture in the nucleus kinetically facilitates local exploration of Sox2, confined Sox 2 target search and thereby facilitated Sox2 activity in the regulation of its target gene expression. 
SPT in 2D of TMR-HaloTag-Sox2 visualized by epi- and BBSP illumination $[44,45]$ in living mESCs revealed two populations of Sox2 molecules with respect to their dwelling time on DNA [72]. Short-lived population $(<1 \mathrm{~s})$ represented non-specific binding of Sox2 to DNA, while long-lived population (>10 s) represented Sox2 molecules bound to their specific DNA target sequences. Multifocus microscopy (MFM) that allows high-resolution instantaneous 3D imaging [91] combined with 3D SPT and kinetic modelling uncovered that Sox 2 and Oct 4 search for its targets by trial-and-error sampling mechanism. This mechanism consisted of 3D diffusion events lasting several seconds and interspersed by brief $(<1 \mathrm{~s})$ non-specific collisions before dwelling at specific target DNA for $>10 \mathrm{~s}$. The in vitro assay in which TMR-HaloTag-Sox2 slide along the DNA in 1D complemented the data from living cells and showed that sliding of Sox2 in 1D along open DNA facilitated its 3D diffusion-dominated search for the target DNA followed by assisted binding of Oct4. Oct 4 then helps to stabilize the interaction of Sox 2 with DNA but has little ability to assist Sox 2 in its target search. Instead, Sox2-assisted binding of Oct4 and subsequent binding of Oct4 to Sox2-DNA complex stabilizes the association of Sox2-Oct4 complex with its target DNA [72]. This detailed analysis facilitated by the recent developments in SMLM are in agreement with the previous notion that the interaction between Sox2 and Oct4 is DNA-dependent, further revealed the hierarchically ordered dynamic search mechanism of the enhancer-binding pluripotency regulators Sox 2 and Oct 4 and mechanistically explains the enhanceosome assembly.

The fact that only a small (3\%) subpopulation of Sox 2 molecules is bound to DNA at any given time [72] makes it impossible to infer the spatial distribution of Sox2 enhancer sites simply from fluorescence fluctuations captured by WF imaging or conventional SRM of fixed cells. Those limitations were overcame when LLS imaging and SPT of JF549-HaloTag-Sox2 was implemented to study the precise 3D distribution of Sox 2 enhancer sites in the whole nucleus of a living mESC [92]. This study revealed that Sox 2 bound enhancers are not uniformly distributed throughout the nucleus but display dramatic clustering behavior and form locally enriched distinct higher density clusters (EnCs) characterized by high density clustering of Sox2. Probing of the spatial relationship between Sox2 EnCs and heterichromatin (HC) marker HP1-GFP that forms non-difraction limited structures in the nucleus revealed that the location of EnCs and HC is only very weakly correlated. Specifically, dual-color WF imaging, mapping of the stable Sox2 EnCs by SPT followed by 2D kernel density estimator and creation of an EnCs intensity map in the nucleus that were superimposed with the HP1-GFP. This allowed for the quantitative pixel-to-pixel correlation between EnCs and HC, which was confirmed by pair cross-correlation analysis (PCC). PCC, which examines the degree of co-clustering and co-localization between two types of molecules [93], showed no apparent spatial correlation between EnC and HC intensity maps [92]. Additionally, LLS imaging and SPT analysis confirmed and further strengthened the notion of spatial separation between Sox2 EnCs and HC in 3D and showed lower levels of Sox 2 in HC compared to surrounding sub-nuclear regions. Finally, dual-color labeling of one target, Halo-Sox2, in the same living mESC with two dyes, JF549 and JF646, and WF imaging of each dye under different conditions, enabled simultaneous visualization of Sox2 EnCs and the dynamics of diffusing and binding Sox 2 molecules. Mapping of stably bound Sox 2 in EnCs was performed by low excitation and long acquisition times of JF-646-HaloTag-Sox2, while tracking of the fast diffusing and binding dynamics of JF549-HaloTag-Sox2 was performed using high excitation power and short acquisition times. This analysis revealed that most of the Sox 2 molecules in EnCs were in a bound state [92].

The single molecule kinetics of steroid receptors, such as glucocorticoid receptor (GR) tagged with PC-FP mEos2 in living MCF-7 cell line was facilitated by RLS microscopy [47]. The diffusional characteristics of mEos2-GR and other TF, estrogen receptor- $\alpha$ was inferred on the principle of detection by localization [94]. Detection by localization does not rely on the detection of long continuous traces and suggested almost a magnitude lower DNA residence time of GR than those earlier obtained by FRAP [95]. This finding 
documents previously unappreciated high dynamics of GR and underscores the advantage of high spatial and temporal resolution of SMLM for studying TF kinetics.

Kinetic studies of yet other TFs, protooncogene c-Myc and elongation factor $\mathrm{P}-\mathrm{TEFb}$, revealed that despite their similar diffusion rates, each TF explores the space differently, highlighting their distinct roles in gene expression [67]. SPT and detailed quantitative analysis of the diffusion kinetics of Dendra2-tagged c-Myc and P-TEFb, visualized by HILO illumination and precisely controlled photoconversion of Dendra2 allowed for the selective visualization of individual Dendra2-tagged c-Myc and P-TEFb and statistical evaluation of their dynamics. Diffusion rates of TFs exceed $10 \mu^{2} / \mathrm{s}$ and therefore the localizations of individual TF molecules deviate from the classical Gaussian PSF. Izeddin et al., devised an algorithm that allowed continuous tracking of TFs diffusing at rates exceeding $10 \mu \mathrm{m}^{2} / \mathrm{s}$. This analysis revealed that although the dynamics of both TFs were similarly restrained by their interaction with nuclear structures, $\mathrm{c}-\mathrm{Myc}$ and P-TEFb adopt different strategies for the exploration of the nuclear space. While Dendra2-tagged c-Myc globally explores the nucleus and diffuses similarly to free Dendra2, individual Dendra2tagged P-TEFb molecules explored the nuclear volume by sampling a space of reduced dimensionality and displaying characteristic of exploration constrained by fractal structures [67].

The discovery of the hierarchical and dynamic assembly mechanism of the general TFs suggests that this previously undetected mechanism of RNAPII pre-initiation is universal [96]. In vitro single molecule imaging studies allowed to study the kinetics of individual purified transcription factors and revealed their stepwise assembly into the preinitiation complex in a defined environment $[97,98]$. The in vitro studies were supported by fast tracking of multiple TFs simultaneously in living cells with high spatiotemporal resolution using high-sensitivity multicamera system with stroboscopic illumination [99]. In living cells, general TFs bound promoter dynamically and transiently and RNAPII preinitiation complex assembled hierarchically [96], similar to the enhanceosome [72].

Taken together, the single molecule imaging uncovered previously unknown Sox2 EnCs positioning throughout the 3D space inside the nucleus and the likelihood of Sox2 finding and binding to EnCs. These detailed quantitative analyses of the Sox 2 dynamics and spatial distribution of Sox 2 EnCs provided valuable insight into the spatial organization and dynamics of the enhancer-binding pluripotency regulators in embryonic stem cells, which greatly extended our mechanistic understanding of the spatial and temporal regulation of gene expression in pluripotent cells and mammalian development at molecular level. Moreover, the exemplar studies reviewed above underscored the advantages of single-molecule imaging and revealed previously undetected hierarchical transient-tostable transition of TF-binding dynamics that regulate the assembly of RNAPII pre-initiation complex. In the next section, we discuss the recent progress in the understanding of RNAPII transcription dynamics on the example of several detailed single molecule imaging studies.

\section{Dynamics of RNAPII by SMLM}

Transcription by RNAPII is essential for gene expression and therefore fundamental for cellular activities. RPB1 enzyme, the large catalytic subunit of RNAPII central of the transcription process, forms spatially compartmentalized foci [100-102]. These foci, earlier visualized by combination of light and cryo-electron microscopy [123] are visible in the chemically fixed cells immunolabeled by anti-RPB1 C-terminal domain (CTD) primary antibody followed by secondary antibody labeled by Alexa Fluor 647 and imaged by dSTORM (Fig. 2). Human RPB1 CTD contains 52 heptad repeats, which can result in the binding of multiple antibodies in the immunolabeling experiments. Tagging of individual RPB1 molecules with a FP or self-labeling tag, such as HaloTag or SNAP tag, represent more accurate approaches. Replacing of endogenous RPB1 in living cells with RPB1 fused to PA-FP Dendra2 or tagging of endogenous RPB1 by CRISPR/Cas9 with Dendra2 or HaloTag and allowed to follow the dynamics of individual RPB1 molecules by SMLM in cell 
lines and embryonic stem cells $[63,65,103]$. The analysis of real-time dynamics of Dendra2-RPB1 in human U-2 OS cells by time-correlated (tc)PALM showed that RPB1 in living cells exhibits transient dynamic clustering clearly distinct from static clusters. This analysis of the temporal sequence of individual RPB1 molecule detections leading to the formation of a particular RPB1 cluster favors the self-organization model of highly dynamic de novo clustering of RPB1 over the deterministic organization of static RPB1 clusters [63]. The half-life of RPB1 clusters was in order of seconds [63], which is significantly faster than several minutes required to complete the transcription of a typical mammalian gene. Further extending the tcPALM analysis to dual-color mode in living mouse embryonic fibroblasts (MEFs) allowed for the simultaneous visualization of RPB1 and nascent mRNA transcript [64, 65]. Simultaneous single-molecule imaging of Dendra2-RPB1 and nascent mRNA containing MS2 hairpin loops recognized by Halo-MCP [64, 104, 105] labeled with Halo-substrate JF646 by tcPALM and STORM, resp., revealed that the number of nascent mRNA molecules directly correlates with the life-time of RPB1 clusters. Transcription activation led to the increased life-time of RPB1 clusters but not of their frequency, which indicates increased loading of RNAPII molecules onto the gene. The quantitative analysis of the single-molecule dynamics of RPB1 clusters and nascent mRNA supported by theoretical modeling is, however, inconsistent with elongating RNAPII, because the time required for production of single mRNA molecule is much longer than the lifetime of RPB1 clusters measured by tcPALM. This notion is nevertheless in agreement with another study that used live-cell SPT of Halo-RPB1 labeled with PA-JF549 and imaged by stroboscopic photoactivation [103]. This study showed that the length of CTD, e.g. the number or heptad repeats, substantially influences RPB1 dynamics. Shorter CTDs, such as 26 heptad repeats present in S. cerevisiae, resulted in the higher RPB1 cluster dynamics and artificial lengthening of RPB1 CTD led to the lower dynamics of RPB1 clusters and their increased association with chromatin [103]. Intriguingly, RPB1 CTD shortening in U2 OS cells delayed transcription activation [106], which is in line with the evidence obtained by SMLM that RPB1 CTD interacts in vivo with TF hubs [106]. Importantly, Boehning and co-workers [103] showed that upon its CTD phosphorylation and switching into the elongation phase, RPB1 clusters dissolve, indicating that elongating RNAPII leaves clusters formed at the (pre-)initiation phase [103]. The notion of the individual RNAPII molecules that transcribe genes and produce mRNA in the elongation phase is in agreement with at least two previous studies. In the first study the authors probed by RLSSRM the spatial organization of RPB1 tagged with SNAP-tag and labelled by TMR SNAPsubstrate [48]. Another study by Bayesian localization microscopy [109] that allowed the direct observation of the assembly and disassembly of Dendra2-RPB1 also revealed that RNAPII clusters on the actively transcribed genes in the pre-elongation phase [108]. Therefore, the dynamic clustering of RPB1 is linked to the assembly of macromolecular complexes during pre-initiation and initiation steps of transcription. Short-lived clusters observed on the actively transcribed gene are transient agglomerations of $~ 80$ polymerases, which are distinct from the much lower counts of elongating polymerases that likely appeared as low frequency of detections [64].

Spatial relationship and inferred functional correlation of RNAPII distribution and Sox2 enhancers was investigated in living mECSs by 2D SPT and mapping of Sox2 EnCs (as described in the previous section) by low-excitation and long-acquisition detection of stable DNA bound JF646-HaloTag-Sox2 molecules followed by PALM imaging of photoconverted Dendra2-RPB1 [63, 92]. Auto-correlation analysis revealed that RPB1 molecules displayed local density fluctuations, e.g. clustering of RPB1 [92], consistent with previous reports [63]. However, RPB1 clusters were more evenly distributed throughout the nucleus and had lower packing densities than highly clustered Sox2 EnCs that also displayed tighter packing [92]. Quantification of the spatial relationship between the distribution patterns of RPB1 and Sox 2 by pixel-to-pixel correlation between the intensity of RPB1 and Sox2 EnCs in individual cells showed that EnC regions are generally correlated with RPB1 occupancy and pair cross-correlation of Sox2 EnCs with RPB1-enriched regions showed significant degree of co-localization/clustering. However, due to the tighter clustering of 
Sox2 enhancers, most Sox 2 EnC regions contained significant levels of RPB1, whereas only a subset of RPB1 enriched regions overlapped with Sox2 EnCs [92], which is in agreement with the previous notion that Sox 2 only targets a subset of genes transcribed in mESCs [122].

The dynamic clustering of RPB1 before productive transcription elongation revealed by tcPALM and SPT analyses has implications for the fine regulation of gene expression and were further confirmed by detailed quantitative analysis of the assembly of macromolecular complexes during pre-initiation and initiation steps of transcription. Transcription coactivator Mediator (Med) assists in the assembly of a transcription pre-initiation complex. Endogenous Med tagged with Dendra2 forms similarly to RPB1 small $(\sim 100 \mathrm{~nm})$ transient (lifetime $\sim 10 \mathrm{~s}$ ) and large $(>300 \mathrm{~nm}$ ) stable (lifetime $>100 \mathrm{~s}$ ) clusters in living mouse embryonic stem cells (mESC). The differentiation of mESCs into epiblast-like cells (EpiLCs) had no apparent effect on the small transient Med clusters but reduced the size and the number of large stable Med clusters [66]. Dual-color lattice light-sheet imaging of JF646-HaloTag-Med followed by imaging of Dendra2-RPB1 in mESCs revealed that the vast majority (90\%) of large stable Med clusters also contained RPB1. The association between Med and RPB1 appeared to be hierarchical with Med clusters serving as a scaffold for the RPB1recruitment. Single-color LLS-SRM of JF646-HaloTag-RPB1 or JF646-HaloTag-Med dynamics that allowed fast (order of seconds) volumetric acquisitions of the whole nucleus in living mESC and precise 4D tracking revealed high mobility and coalescence of individual RPB1 or Med clusters into larger condensates. Moreover, dual-color imaging of JF549-HaloTag-Med together with a gene loci visualized by mRNA-incorporated MS2 hairpin that binds MCP-HaloTag-JF646 [64, 104, 105] revealed the mobility and coalescence of individual RPB1 or Med clusters and transient co-localization of Med with the gene loci during transcription pre-initiation [66]. Taken together, LLS-SRM [92] that allows gentle multicolor 4D imaging of individual molecule dynamics in living cells helped to elaborate on the previous measurements of single molecule dynamics by tcPALM. These individual techniques separately revealed the induced condensation of Med at enhancers followed by recruitment of RNAPII and preceding mRNA production [64], which is consistent with the dynamic recruitment of Sox 2 and Oct 4 to their enhancers $[66,92]$ that we discussed in the preceding section.

In summary, several reviewed SMLM approaches enabled to study the gene regulation in unprecedented spatial and temporal details and thereby helped to finely refine our mechanistic understanding of the regulation of gene expression. Integration of the information obtained by the SMLM together with biochemistry helped to build a mechanistic model of the localized hierarchical recruitment of co-activators and TFs during the preinitiation phase followed by the recruitment and formation of RNAPII clusters during the initiation phase and followed by RNAPII leaving the clusters during elongation phase. This hierarchical and dynamic principle of the gene expression regulation and the discovery of RNAPII forming transient condensates that dictates the productive transcriptional output progressively changed our mechanistic understanding of gene expression.

\section{Conclusions and Perspectives}

Since the publication in 2010 of one of the seminal reviews on SRM [11] twelve years passed during which the expectation of authors that "it will still take time and further engineering until [the] technical developments [of SMLM] find their way into commercial systems" became reality. Moreover, as we have reviewed here on the example of some of the SMLM applications to monitor the dynamics of TFs and RNAPII in living cell, in the past decade we have witnessed the rapid progress in the 4D single molecule kinetic studies in living cells. Nevertheless, many areas of the cell nucleus that hold secrets of the gene expression mechanism that lead to the establishment and propagation of the living matter in space and time remain unexplored. We believe that relatively new but rapidly growing field of nuclear lipid biology will greatly benefit from the progressive development of the quantitative single molecule imaging approaches. Nuclear lipids and phosphatidylinositol phosphates (PIPs) in particular, form small foci variably distributed in the eukaryotic 
cell nucleus (Fig. 3), localized to the specific sub-nuclear compartments and linked with the crucial nuclear processes [110-118]. So far, we started to paint with nanometer precision the static pictures of nuclear PIP distributions [119]. However, as we learned from the cytoplasmic membrane, intracellular membrane compartments and from the nuclear envelope, lipids are highly mobile entities [52]. Although a variety of tools is applicable for the specific visualization of various lipid species in the membranes, lipids in the membranes display 2D distributions and dynamics that differ from the nuclear interior that is organized within membrane-less sub-compartments [120]. Therefore, development of the tools for visualization of specific nuclear lipids by single-molecule imaging together with the use of novel fluorophores and implementation of the 3D SPT will be crucial for the kinetic studies of the nuclear lipids and their roles in the establishment of the functional nuclear architecture and in gene expression. Tracking of the individual molecules of specific lipid probes in the compact nuclear environment will be guided by the SPT of transcription factors. Combination of SMLM with EM provided valuable contextual information about cellular nanostructures $[24,121]$ and hold a potential for unraveling of the lipid, and in particular PIP, identity of the nuclear gene expression compartments.
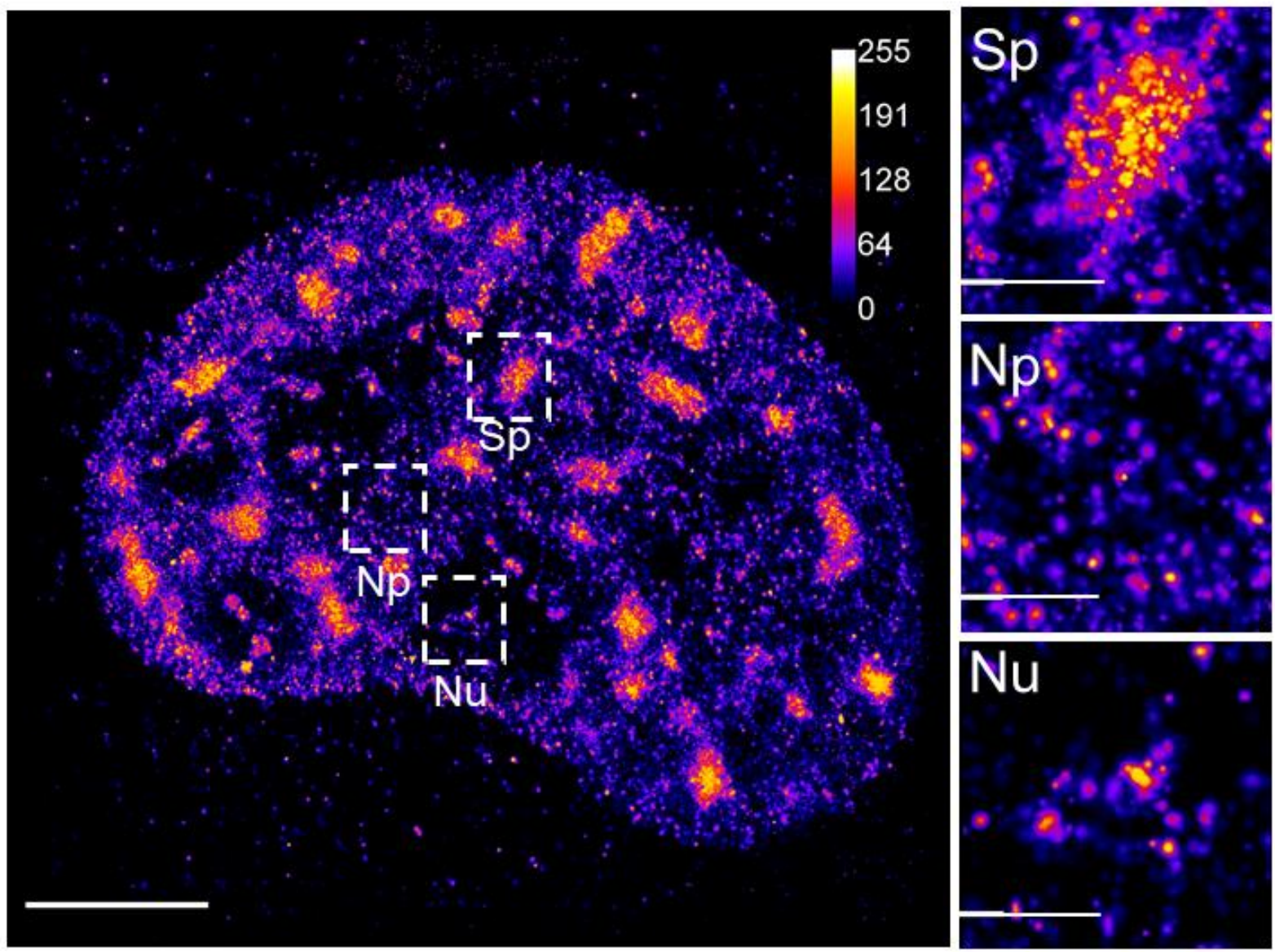

Figure 3. 3D dSTORM imaging of nuclear phosphatidylinositol 4,5-bisphosphate. A 2D projection of the 3D dSTORM image of the nuclear phosphatidylinositol 4,5-bisphosphate (PIP2) indirectly immunolabeled by primary anti-PIP2 antibody followed by secondary antibody conjugated with Alexa Fluor 647 and imaged by 3D dSTORM. Selected regions show specific pattern of PIP2 associated with nuclear speckles (Sp), nucleoplasm (Np) and nucleolus (Nu). Bar $=5 \mu \mathrm{m}$; inset 1 $\mu \mathrm{m}$.

Supplementary Materials: The following are available online at www.mdpi.com/xxx/s1, Figure S1: title, Table S1: title, Video S1: title.

Author Contributions: Pe. H. conceptualization, investigation, methodology, analysis, data curation, validation, visualization, manuscript writing; O. Š. conceptualization, investigation, software, 
visualization, manuscript writing; Pa. H. supervision, funding, conceptualization, investigation, manuscript writing.

Funding: We acknowledge the Light Microscopy Core Facility, IMG CAS, Prague, Czech Republic, supported by grants "National Infrastructure for Biological and Medical Imaging" (MEYS LM2018129), "Modernization of the national infrastructure for biological and medical imaging Czech-BioImaging" (MEYS - CZ.02.1.01/0.0/0.0/18_046/0016045) and formal National Program of Sustainability NPUI LO1220 and LO1419 (RVO: 68378050-KAV-NPUI); Laboratory of Confocal and Fluorescence Microscopy co-financed by the ERDF and the state budget of the Czech Republic, projects no. CZ.1.05/4.1.00/16.0347 and CZ.2.16/3.1.00/21515, and supported by the Czech-BioImaging large RI project LM2018129. Imaging Methods Core Facility at BIOCEV, institution supported by the MEYS CR (Large RI Project LM2018129 Czech-BioImaging) and ERDF (project No. CZ.02.1.01/0.0/0.0/16_013/0001775) for their support with the imaging presented herein; Grant Agency of the Czech Republic (Grant nos. 19-05608S and 18-19714S); by the Czech Academy of Sciences (Grant no. JSPS-20-06); Institutional Research Concept of the Institute of Molecular Genetics (Grant no. RVO: 68378050); MEYS CR (COST Inter-excellence internship LTC19048, LTC20024 and Action 15214 EuroCellnet).

Acknowledgments: We are grateful to Katarína Mrvová for the poinilistic artistic illustration presented in Figure 3 and we would like to thank to Ivan Novotny from the Light Microscopy Core Facility, IMG CAS, Prague, Czech Republic, for his help with imaging presented in Figure 1 and to Marie Olšinová from the Imaging Methods Core Facility at BIOCEV, Vestec, Czech Republic, for her help with imaging presented in Figure 3; to Lenka Pišlová for her excellent administrative assistance, to Iva Jelínková for her assistance with the cell cultures, Pavel Kř́íz for the lab management and Martin Sztacho for discussions.

Conflicts of Interest: "The authors declare no conflict of interest."

\section{References}

1. Abbe E. Beiträge zur Theorie des Mikroskops und der mikroskopischen Wahrnehmung. Archiv f. mikrosk. Anatomie 1873;9:413-468. doi: 10.1007/BF02956173_

2. Cremer C, Szczurek A, Schock F, Gourram A, Birk U. Super-resolution microscopy approaches to nuclear nanostructure imaging. Methods. 2017;123:11-32. doi:10.1016/j.ymeth.2017.03.019

3. Rayleig, L. On the Theory of Optical Images, with special reference to the Microscope. Journal of the Royal Microscopical Society 1896;23:474-482. doi: 10.1111/j.1365-2818.1903.tb04831.x

4. Pombo A, Hollinshead M, Cook PR. Bridging the resolution gap: Imaging the same transcription factories in cryosections by light and electron microscopy. J Histochem Cytochem. 1999 Apr;47(4):471-80. doi: 10.1177/002215549904700405. PMID: 10082748.

5. Niedojadlo J, Perret-Vivancos C, Kalland KH, et al. Transcribed DNA is preferentially located in the perichromatin region of mammalian cell nuclei. Exp Cell Res. 2011;317(4):433-444. doi:10.1016/j.yexcr.2010.10.026

6. Rouquette J, Cremer C, Cremer T, Fakan S. Functional nuclear architecture studied by microscopy: present and future. Int Rev Cell Mol Biol. 2010;282:1-90. doi:10.1016/S1937-6448(10)82001-5

7. Hanske J, Sadian Y, Müller CW. The cryo-EM resolution revolution and transcription complexes. Curr Opin Struct Biol. 2018;52:8-15. doi:10.1016/j.sbi.2018.07.002

8. Hell SW. Far-field optical nanoscopy. Science. 2007;316(5828):1153-1158. doi:10.1126/science.1137395

9. Hell SW. Microscopy and its focal switch. Nat Methods. 2009 Jan;6(1):24-32. doi: 10.1038/nmeth.1291. PMID: 19116611.

10. Hell SW. Toward fluorescence nanoscopy. Nat Biotechnol. 2003;21(11):1347-1355. doi:10.1038/nbt895

11. Schermelleh L, Heintzmann R, Leonhardt H. A guide to super-resolution fluorescence microscopy. J Cell Biol. 2010;190(2):165-175. doi:10.1083/jcb.201002018

12. Tam J, Merino D. Stochastic optical reconstruction microscopy (STORM) in comparison with stimulated emission depletion (STED) and other imaging methods. J Neurochem. 2015;135(4):643-658. doi:10.1111/jnc.13257 
13. Sahl SJ, Hell SW. High-Resolution 3D Light Microscopy with STED and RESOLFT. In: Bille JF, ed. High Resolution Imaging in Microscopy and Ophthalmology: New Frontiers in Biomedical Optics. Cham (CH): Springer; August 14, 2019.3-32.

14. Hofmann M, Eggeling C, Jakobs S, Hell SW. Breaking the diffraction barrier in fluorescence microscopy at low light intensities by using reversibly photoswitchable proteins. Proc Natl Acad Sci U S A. 2005;102(49):17565-17569. doi:10.1073/pnas.0506010102

15. Gustafsson MG. Nonlinear structured-illumination microscopy: wide-field fluorescence imaging with theoretically unlimited resolution. Proc Natl Acad Sci U S A. 2005;102(37):13081-13086. doi:10.1073/pnas.0406877102

16. Gustafsson MG. Surpassing the lateral resolution limit by a factor of two using structured illumination microscopy. $J \mathrm{Mi}$ crosc. 2000;198(Pt 2):82-87. doi:10.1046/j.1365-2818.2000.00710.x

17. Bailey B, Farkas DL, Taylor DL, Lanni F. Enhancement of axial resolution in fluorescence microscopy by standing-wave excitation. Nature. 1993;366(6450):44-48. doi:10.1038/366044a0

18. Gustafsson MG, Shao L, Carlton PM, et al. Three-dimensional resolution doubling in wide-field fluorescence microscopy by structured illumination. Biophys J. 2008;94(12):4957-4970. doi:10.1529/biophysj.107.120345

19. Schermelleh L, Carlton PM, Haase S, et al. Subdiffraction multicolor imaging of the nuclear periphery with 3D structured illumination microscopy. Science. 2008;320(5881):1332-1336. doi:10.1126/science.1156947

20. Baddeley D, Chagin VO, Schermelleh L, et al. Measurement of replication structures at the nanometer scale using superresolution light microscopy. Nucleic Acids Res. 2010;38(2):e8. doi:10.1093/nar/gkp901

21. Kner P, Chhun BB, Griffis ER, Winoto L, Gustafsson MG. Super-resolution video microscopy of live cells by structured illumination. Nat Methods. 2009;6(5):339-342. doi:10.1038/nmeth.1324

22. Klar TA, Jakobs S, Dyba M, Egner A, Hell SW. Fluorescence microscopy with diffraction resolution barrier broken by stimulated emission. Proc Natl Acad Sci U S A. 2000;97(15):8206-8210. doi:10.1073/pnas.97.15.8206

23. Hell SW, Wichmann J. Breaking the diffraction resolution limit by stimulated emission: stimulated-emission-depletion fluorescence microscopy. Opt Lett. 1994;19(11):780-782. doi:10.1364/ol.19.000780

24. Betzig E, Patterson GH, Sougrat R, et al. Imaging intracellular fluorescent proteins at nanometer resolution. Science. 2006;313(5793):1642-1645. doi:10.1126/science.1127344

25. Hess ST, Girirajan TP, Mason MD. Ultra-high resolution imaging by fluorescence photoactivation localization microscopy. Biophys J. 2006;91(11):4258-4272. doi:10.1529/biophysj.106.091116

26. Rust MJ, Bates M, Zhuang X. Sub-diffraction-limit imaging by stochastic optical reconstruction microscopy (STORM). Nat Methods. 2006;3(10):793-795. doi:10.1038/nmeth929

27. Heilemann M, van de Linde S, Schüttpelz M, et al. Subdiffraction-resolution fluorescence imaging with conventional fluorescent probes. Angew Chem Int Ed Engl. 2008;47(33):6172-6176. doi:10.1002/anie.200802376

28. Wombacher R, Heidbreder M, van de Linde S, et al. Live-cell super-resolution imaging with trimethoprim conjugates. Nat Methods. 2010;7(9):717-719. doi:10.1038/nmeth.1489

29. van de Linde S, Löschberger A, Klein T, et al. Direct stochastic optical reconstruction microscopy with stand ard fluorescent probes. Nat Protoc. 2011;6(7):991-1009. Published 2011 Jun 16. doi:10.1038/nprot.2011.336

30. Lippincott-Schwartz J, Patterson GH. Photoactivatable fluorescent proteins for diffraction-limited and super-resolution imaging. Trends Cell Biol. 2009;19(11):555-565. doi:10.1016/j.tcb.2009.09.003

31. Fernández-Suárez M, Ting AY. Fluorescent probes for super-resolution imaging in living cells. Nat Rev Mol Cell Biol. 2008;9(12):929-943. doi:10.1038/nrm2531

32. Lidke K, Rieger B, Jovin T, Heintzmann R. Superresolution by localization of quantum dots using blinking statistics. Opt Express. 2005;13(18):7052-7062. doi:10.1364/opex.13.007052

33. Huang B, Jones SA, Brandenburg B, Zhuang X. Whole-cell 3D STORM reveals interactions between cellular structures with nanometer-scale resolution. Nat Methods. 2008;5(12):1047-1052. doi:10.1038/nmeth.1274 
34. Huang B, Wang W, Bates M, Zhuang X. Three-dimensional super-resolution imaging by stochastic optical reconstruction microscopy. Science. 2008;319(5864):810-813. doi:10.1126/science.1153529

35. Jones SA, Shim SH, He J, Zhuang X. Fast, three-dimensional super-resolution imaging of live cells. Nat Methods. 2011;8(6):499-508. doi:10.1038/nmeth.1605

36. Legant WR, Shao L, Grimm JB, et al. High-density three-dimensional localization microscopy across large volumes. Nat Methods. 2016;13(4):359-365. doi:10.1038/nmeth.3797

37. Juette MF, Gould TJ, Lessard MD, et al. Three-dimensional sub-100 nm resolution fluorescence microscopy of thick samples. Nat Methods. 2008;5(6):527-529. doi:10.1038/nmeth.1211

38. Pavani SR, Thompson MA, Biteen JS, et al. Three-dimensional, single-molecule fluorescence imaging beyond the diffraction limit by using a double-helix point spread function. Proc Natl Acad Sci U S A. 2009;106(9):2995-2999. doi:10.1073/pnas.0900245106

39. Liu Z, Lavis LD, Betzig E. Imaging live-cell dynamics and structure at the single-molecule level. Mol Cell. 2015;58(4):644659. doi:10.1016/j.molcel.2015.02.033

40. Axelrod D. Total internal reflection fluorescence microscopy in cell biology. Traffic. 2001;2(11):764-774. doi:10.1034/j.16000854.2001.21104.x

41. Tokunaga M, Imamoto N, Sakata-Sogawa K. Highly inclined thin illumination enables clear single-molecule imaging in cells [published correction appears in Nat Methods. 2008 May;5(5):455]. Nat Methods. 2008;5(2):159-161. doi:10.1038/nmeth1171

42. Huisken J, Swoger J, Del Bene F, Wittbrodt J, Stelzer EH. Optical sectioning deep inside live embryos by selective plane illumination microscopy. Science. 2004;305(5686):1007-1009. doi:10.1126/science.1100035

43. Hoyer P, de Medeiros G, Balázs B, et al. Breaking the diffraction limit of light-sheet fluorescence microscopy by RESOLFT. Proc Natl Acad Sci U S A. 2016;113(13):3442-3446. doi:10.1073/pnas.1522292113

44. Planchon TA, Gao L, Milkie DE, et al. Rapid three-dimensional isotropic imaging of living cells using Bessel beam plane illumination. Nat Methods. 2011;8(5):417-423. doi:10.1038/nmeth.1586

45. Gao L, Shao L, Chen BC, Betzig E. 3D live fluorescence imaging of cellular dynamics using Bessel beam plane illumination microscopy. Nat Protoc. 2014;9(5):1083-1101. doi:10.1038/nprot.2014.087

46. Chen BC, Legant WR, Wang K, et al. Lattice light-sheet microscopy: imaging molecules to embryos at high spatiotemporal resolution. Science. 2014;346(6208):1257998. doi:10.1126/science.1257998

47. Gebhardt JC, Suter DM, Roy R, et al. Single-molecule imaging of transcription factor binding to DNA in live mammalian cells. Nat Methods. 2013;10(5):421-426. doi:10.1038/nmeth.2411

48. Zhao ZW, Roy R, Gebhardt JC, Suter DM, Chapman AR, Xie XS. Spatial organization of RNA polymerase II inside a mammalian cell nucleus revealed by reflected light-sheet superresolution microscopy. Proc Natl Acad Sci U S A. 2014;111(2):681686. doi:10.1073/pnas.1318496111

49. Balzarotti F, Eilers Y, Gwosch KC, et al. Nanometer resolution imaging and tracking of fluorescent molecules with minimal photon fluxes. Science. 2017;355(6325):606-612. doi:10.1126/science.aak9913

50. Eilers Y, Ta H, Gwosch KC, Balzarotti F, Hell SW. MINFLUX monitors rapid molecular jumps with superior spatiotemporal resolution. Proc Natl Acad Sci U S A. 2018;115(24):6117-6122. doi:10.1073/pnas.1801672115

51. Gwosch KC, Pape JK, Balzarotti F, Hoess P, Ellenberg J, Ries J, Hell SW. MINFLUX nanoscopy delivers 3D multicolor nanometer resolution in cells. Nat Methods. 2020 Feb;17(2):217-224. doi: 10.1038/s41592-019-0688-0. Epub 2020 Jan 13. PMID: 31932776.

52. Schmidt R, Weihs T, Wurm CA, et al. MINFLUX nanometer-scale 3D imaging and microsecond-range tracking on a common fluorescence microscope. Nat Commun. 2021;12(1):1478. Published 2021 Mar 5. doi:10.1038/s41467-021-21652-z

53. Dempsey GT, Vaughan JC, Chen KH, Bates M, Zhuang X. Evaluation of fluorophores for optimal performance in localization-based super-resolution imaging. Nat Methods. 2011;8(12):1027-1036. Published 2011 Nov 6. doi:10.1038/nmeth.1768 
54. Grimm JB, Tkachuk AN, Xie L, et al. A general method to optimize and functionalize red-shifted rhodamine dyes. Nat Methods. 2020;17(8):815-821. doi:10.1038/s41592-020-0909-6

55. Lardon N, Wang L, Tschanz A, Hoess P, Tran M, D’Este E, et al. Systematic Tuning of Rhodamine Spirocyclization for Super-Resolution Microscopy. bioRxiv. 2021:2021.05.20.444797. doi: 10.1101/2021.05.20.444797.

56. Wiedenmann J, Ivanchenko S, Oswald F, et al. EosFP, a fluorescent marker protein with UV-inducible green-to-red fluorescence conversion. Proc Natl Acad Sci U S A. 2004;101(45):15905-15910. doi:10.1073/pnas.0403668101

57. McKinney SA, Murphy CS, Hazelwood KL, Davidson MW, Looger LL. A bright and photostable photoconvertible fluorescent protein. Nat Methods. 2009;6(2):131-133. doi:10.1038/nmeth.1296

58. Gurskaya NG, Verkhusha VV, Shcheglov AS, et al. Engineering of a monomeric green-to-red photoactivatable fluorescent protein induced by blue light. Nat Biotechnol. 2006;24(4):461-465. doi:10.1038/nbt1191

59. Chudakov DM, Lukyanov S, Lukyanov KA. Tracking intracellular protein movements using photoswitchable fluorescent proteins PS-CFP2 and Dendra2. Nat Protoc. 2007;2(8):2024-2032. doi:10.1038/nprot.2007.291

60. Zhang L, Gurskaya NG, Merzlyak EM, et al. Method for real-time monitoring of protein degradation at the single cell level. Biotechniques. 2007;42(4):446-450. doi:10.2144/000112453

61. Lee SH, Shin JY, Lee A, Bustamante C. Counting single photoactivatable fluorescent molecules by photoactivated localization microscopy (PALM). Proc Natl Acad Sci U S A. 2012;109(43):17436-17441. doi:10.1073/pnas.1215175109

62. Landgraf D, Okumus B, Chien P, Baker TA, Paulsson J. Segregation of molecules at cell division reveals native protein localization. Nat Methods. 2012;9(5):480-482. Published 2012 Apr 8. doi:10.1038/nmeth.1955

63. Cisse II, Izeddin I, Causse SZ, et al. Real-time dynamics of RNA polymerase II clustering in live human cells. Science. 2013;341(6146):664-667. doi:10.1126/science.1239053

64. Cho WK, Jayanth N, English BP, et al. RNA Polymerase II cluster dynamics predict mRNA output in living cells. Elife. 2016;5:e13617. Published 2016 May 3. doi:10.7554/eLife.13617

65. Cho WK, Jayanth N, Mullen S, Tan TH, Jung YJ, Cissé II. Super-resolution imaging of fluorescently labeled, endogenous RNA Polymerase II in living cells with CRISPR/Cas9-mediated gene editing. Sci Rep. 2016;6:35949. Published 2016 Oct 26. doi:10.1038/srep35949

66. Cho WK, Spille JH, Hecht M, et al. Mediator and RNA polymerase II clusters associate in transcription-dependent condensates. Science. 2018;361(6400):412-415. doi:10.1126/science.aar4199

67. Izeddin I, Récamier V, Bosanac L, et al. Single-molecule tracking in live cells reveals distinct target-search strategies of transcription factors in the nucleus. Elife. 2014;3:e02230. Published 2014 Jun 12. doi:10.7554/eLife.02230

68. Rollins GC, Shin JY, Bustamante C, Pressé S. Stochastic approach to the molecular counting problem in superresolution microscopy. Proc Natl Acad Sci U S A. 2015;112(2):E110-E118. doi:10.1073/pnas.1408071112

69. Gautier A, Juillerat A, Heinis C, et al. An engineered protein tag for multiprotein labeling in living cells. Chem Biol. 2008;15(2):128-136. doi:10.1016/j.chembiol.2008.01.007

70. Keppler A, Gendreizig S, Gronemeyer T, Pick H, Vogel H, Johnsson K. A general method for the covalent labeling of fusion proteins with small molecules in vivo. Nat Biotechnol. 2003;21(1):86-89. doi:10.1038/nbt765

71. Los GV, Encell LP, McDougall MG, et al. HaloTag: a novel protein labeling technology for cell imaging and protein analysis. ACS Chem Biol. 2008;3(6):373-382. doi:10.1021/cb800025k

72. Chen J, Zhang Z, Li L, et al. Single-molecule dynamics of enhanceosome assembly in embryonic stem cells. Cell. 2014;156(6):1274-1285. doi:10.1016/j.cell.2014.01.062

73. Presman DM, Ball DA, Paakinaho V, et al. Quantifying transcription factor binding dynamics at the single-molecule level in live cells. Methods. 2017;123:76-88. doi:10.1016/j.ymeth.2017.03.014

74. Grimm JB, English BP, Chen J, et al. A general method to improve fluorophores for live-cell and single-molecule microscopy. Nat Methods. 2015;12(3):244-250. doi:10.1038/nmeth.3256 
75. Zheng Q, Ayala AX, Chung I, et al. Rational Design of Fluorogenic and Spontaneously Blinking Labels for Super-Resolution Imaging [published correction appears in ACS Cent Sci. 2020 Oct 28;6(10):1844]. ACS Cent Sci. 2019;5(9):1602-1613. doi:10.1021/acscentsci.9b00676

76. Grimm JB, Muthusamy AK, Liang Y, et al. A general method to fine-tune fluorophores for live-cell and in vivo imaging. Nat Methods. 2017;14(10):987-994. doi:10.1038/nmeth.4403

77. Grimm JB, English BP, Choi H, et al. Bright photoactivatable fluorophores for single-molecule imaging. Nat Methods. 2016;13(12):985-988. doi:10.1038/nmeth.4034

78. Liu H, Dong P, Ioannou MS, et al. Visualizing long-term single-molecule dynamics in vivo by stochastic protein labeling. Proc Natl Acad Sci U S A. 2018;115(2):343-348. doi:10.1073/pnas.1713895115

79. Binns TC, Ayala AX, Grimm JB, et al. Rational Design of Bioavailable Photosensitizers for Manipulation and Imaging of Biological Systems. Cell Chem Biol. 2020;27(8):1063-1072.e7. doi:10.1016/j.chembiol.2020.07.001

80. Tanenbaum ME, Gilbert LA, Qi LS, Weissman JS, Vale RD. A protein-tagging system for signal amplification in gene expression and fluorescence imaging. Cell. 2014;159(3):635-646. doi:10.1016/j.cell.2014.09.039

81. Gorski SA, Dundr M, Misteli T. The road much traveled: trafficking in the cell nucleus. Curr Opin Cell Biol. 2006;18(3):284290. doi:10.1016/j.ceb.2006.03.002

82. Hager GL, McNally JG, Misteli T. Transcription dynamics. Mol Cell. 2009;35(6):741-753. doi:10.1016/j.molcel.2009.09.005

83. Woringer M, Darzacq X. Protein motion in the nucleus: from anomalous diffusion to weak interactions. Biochem Soc Trans. 2018;46(4):945-956. doi:10.1042/BST20170310

84. McNally JG, Müller WG, Walker D, Wolford R, Hager GL. The glucocorticoid receptor: rapid exchange with regulatory sites in living cells. Science. 2000;287(5456):1262-1265. doi:10.1126/science.287.5456.1262

85. Misteli T. Protein dynamics: implications for nuclear architecture and gene expression. Science. 2001;291(5505):843-847. doi:10.1126/science.291.5505.843

86. Phair RD, Misteli T. Kinetic modelling approaches to in vivo imaging. Nat Rev Mol Cell Biol. 2001;2(12):898-907. doi: $10.1038 / 35103000$

87. Darzacq X, Yao J, Larson DR, et al. Imaging transcription in living cells. Annu Rev Biophys. 2009;38:173-196. doi:10.1146/annurev.biophys.050708.133728

88. Fuda NJ, Ardehali MB, Lis JT. Defining mechanisms that regulate RNA polymerase II transcription in vivo. Nature. 2009;461(7261):186-192. doi:10.1038/nature08449

89. Manley S, Gillette JM, Lippincott-Schwartz J. Single-particle tracking photoactivated localization microscopy for mapping single-molecule dynamics. Methods Enzymol. 2010;475:109-120. doi:10.1016/S0076-6879(10)75005-9

90. Hansen AS, Woringer M, Grimm JB, Lavis LD, Tjian R, Darzacq X. Robust model-based analysis of single-particle tracking experiments with Spot-On. Elife. 2018;7:e33125. Published 2018 Jan 4. doi:10.7554/eLife.33125

91. Abrahamsson S, Chen J, Hajj B, et al. Fast multicolor 3D imaging using aberration-corrected multifocus microscopy. Nat Methods. 2013;10(1):60-63. doi:10.1038/nmeth.2277

92. Liu Z, Legant WR, Chen BC, et al. 3D imaging of Sox2 enhancer clusters in embryonic stem cells. Elife. 2014;3:e04236. Published 2014 Dec 24. doi:10.7554/eLife.04236

93. Veatch SL, Machta BB, Shelby SA, Chiang EN, Holowka DA, Baird BA. Correlation functions quantify super-resolution images and estimate apparent clustering due to over-counting. PLoS One. 2012;7(2):e31457. doi:10.1371/journal.pone.0031457

94. Elf J, Li GW, Xie XS. Probing transcription factor dynamics at the single-molecule level in a living cell. Science. 2007;316(5828):1191-1194. doi:10.1126/science.1141967

95. Sprague BL, Müller F, Pego RL, Bungay PM, Stavreva DA, McNally JG. Analysis of binding at a single spatially localized cluster of binding sites by fluorescence recovery after photobleaching. Biophys J. 2006;91(4):1169-1191. doi:10.1529/biophysj.105.073676 
96. Zhang Z, English BP, Grimm JB, et al. Rapid dynamics of general transcription factor TFIIB binding during preinitiation complex assembly revealed by single-molecule analysis. Genes Dev. 2016;30(18):2106-2118. doi:10.1101/gad.285395.116

97. Zhang Z, Revyakin A, Grimm JB, Lavis LD, Tjian R. Single-molecule tracking of the transcription cycle by sub-second RNA detection. Elife. 2014;3:e01775. doi:10.7554/eLife.01775

98. Revyakin A, Zhang Z, Coleman RA, et al. Transcription initiation by human RNA polymerase II visualized at single-molecule resolution. Genes Dev. 2012;26(15):1691-1702. doi:10.1101/gad.194936.112

99. English BP, Singer RH. A three-camera imaging microscope for high-speed single-molecule tracking and super-resolution imaging in living cells. Proc SPIE Int Soc Opt Eng. 2015;9550:955008. doi:10.1117/12.2190246

100. Jackson DA, Hassan AB, Errington RJ, Cook PR. Visualization of focal sites of transcription within human nuclei. EMBO J. 1993;12(3):1059-1065.

101. Papantonis A, Cook PR. Transcription factories: genome organization and gene regulation. Chem Rev. 2013;113(11):86838705. doi:10.1021/cr300513p

102. Cook PR. A model for all genomes: the role of transcription factories. J Mol Biol. 2010;395(1):1-10. doi:10.1016/j.jmb.2009.10.031

103. Boehning M, Dugast-Darzacq C, Rankovic M, et al. RNA polymerase II clustering through carboxy-terminal domain phase separation. Nat Struct Mol Biol. 2018;25(9):833-840. doi:10.1038/s41594-018-0112-y

104. Bertrand E, Chartrand P, Schaefer M, Shenoy SM, Singer RH, Long RM. Localization of ASH1 mRNA particles in living yeast. Mol Cell. 1998;2(4):437-445. doi:10.1016/s1097-2765(00)80143-4

105. Spille JH, Hecht M, Grube V, Cho WK, Lee C, Cissé II. A CRISPR/Cas9 platform for MS2-labelling of single mRNA in live stem cells. Methods. 2019;153:35-45. doi:10.1016/j.ymeth.2018.09.004

106. Sawicka A, Villamil G, Lidschreiber M, et al. Transcription activation depends on the length of the RNA polymerase II Cterminal domain. EMBO J. 2021;40(9):e107015. doi:10.15252/embj.2020107015

107. Chong S, Dugast-Darzacq C, Liu Z, et al. Imaging dynamic and selective low-complexity domain interactions that control gene transcription. Science. 2018;361(6400):eaar2555. doi:10.1126/science.aar2555

108. Chen X, Wei M, Zheng MM, et al. Study of RNA Polymerase II Clustering inside Live-Cell Nuclei Using Bayesian Nanoscopy [published correction appears in ACS Nano. 2016 Apr 26;10 (4):4882]. ACS Nano. 2016;10(2):2447-2454. doi:10.1021/acsnano.5b07257

109. Cox S, Rosten E, Monypenny J, et al. Bayesian localization microscopy reveals nanoscale podosome dynamics. Nat Methods. 2011;9(2):195-200. Published 2011 Dec 4. doi:10.1038/nmeth.1812

110. Bunce MW, Bergendahl K, Anderson RA. Nuclear PI(4,5)P(2): a new place for an old signal. Biochim Biophys Acta. 2006;1761(5-6):560-569. doi:10.1016/j.bbalip.2006.03.002

111. Lewis AE, Sommer L, Arntzen MØ, et al. Identification of nuclear phosphatidylinositol 4,5-bisphosphate-interacting proteins by neomycin extraction. Mol Cell Proteomics. 2011;10(2):M110.003376. doi:10.1074/mcp.M110.003376

112. Sobol M, Krausová A, Yildirim S, et al. Nuclear phosphatidylinositol 4,5-bisphosphate islets contribute to efficient RNA polymerase II-dependent transcription. J Cell Sci. 2018;131(8):jcs211094. Published 2018 Apr 13. doi:10.1242/jcs.211094

113. Fáberová V, Kalasová I, Krausová A, Hozák P. Super-Resolution Localisation of Nuclear PI(4)P and Identification of Its Interacting Proteome. Cells. 2020;9(5):1191. Published 2020 May 11. doi:10.3390/cells9051191

114. Shah ZH, Jones DR, Sommer L, et al. Nuclear phosphoinositides and their impact on nuclear functions. FEBS J. 2013;280(24):6295-6310. doi:10.1111/febs.12543

115. Cocco L, Follo MY, Manzoli L, Suh PG. Phosphoinositide-specific phospholipase C in health and disease. J Lipid Res. 2015;56(10):1853-1860. doi:10.1194/j1r.R057984

116. Sztacho M, Sobol M, Balaban C, Escudeiro Lopes SE, Hozák P. Nuclear phosphoinositides and phase separation: Important players in nuclear compartmentalization. Adv Biol Regul. 2019;71:111-117. doi:10.1016/j.jbior.2018.09.009 
117. Castano E, Yildirim S, Fáberová V, et al. Nuclear Phosphoinositides-Versatile Regulators of Genome Functions. Cells. 2019;8(7):649. Published 2019 Jun 28. doi:10.3390/cells8070649

118. Sztacho M, Šalovská B, Červenka J, Balaban C, Hoboth P, Hozák P. Limited Proteolysis-Coupled Mass Spectrometry Identifies Phosphatidylinositol 4,5-Bisphosphate Effectors in Human Nuclear Proteome. Cells. 2021;10(1):68. Published 2021 Jan 4. doi:10.3390/cells10010068

119. Hoboth P, Sztacho M, Šebesta O, Schätz M, Castano E, Hozák P. Nanoscale mapping of nuclear phosphatidylinositol phosphate landscape by dual-color dSTORM. Biochim Biophys Acta Mol Cell Biol Lipids. 2021;1866(5):158890. doi:10.1016/j.bbalip.2021.158890

120. Feric M, Vaidya N, Harmon TS, et al. Coexisting Liquid Phases Underlie Nucleolar Subcompartments. Cell. 2016;165(7):1686-1697. doi:10.1016/j.cell.2016.04.047

121. Franke C, Repnik U, Segeletz S, et al. Correlative single-molecule localization microscopy and electron tomography reveals endosome nanoscale domains. Traffic. 2019;20(8):601-617. doi:10.1111/tra.12671

122. Chen $X, X u H$, Yuan $P$, et al. Integration of external signaling pathways with the core transcriptional network in embryonic stem cells. Cell. 2008;133(6):1106-1117. doi:10.1016/j.cell.2008.04.043 